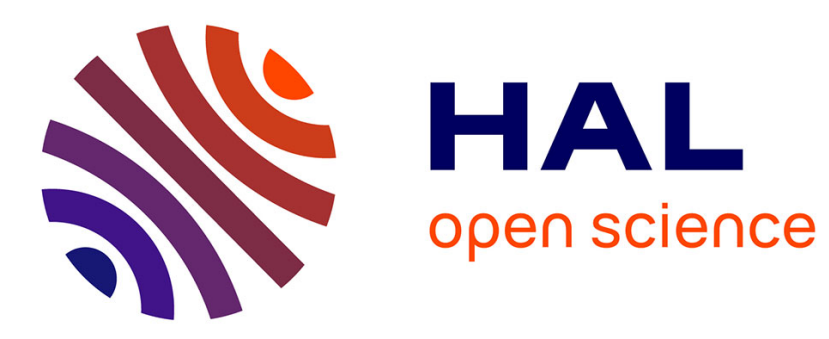

\title{
On constitutive inequalities in nonlinear theory of elastic shells
}

Victor A. Eremeyev, Leonid M. Zubov

\section{To cite this version:}

Victor A. Eremeyev, Leonid M. Zubov. On constitutive inequalities in nonlinear theory of elastic shells. Journal of Applied Mathematics and Mechanics / Zeitschrift für Angewandte Mathematik und Mechanik, 2007, 87 (2), pp.94-101. hal-00835648

\section{HAL Id: hal-00835648 https://hal.science/hal-00835648}

Submitted on 19 Jun 2013

HAL is a multi-disciplinary open access archive for the deposit and dissemination of scientific research documents, whether they are published or not. The documents may come from teaching and research institutions in France or abroad, or from public or private research centers.
L'archive ouverte pluridisciplinaire HAL, est destinée au dépôt et à la diffusion de documents scientifiques de niveau recherche, publiés ou non, émanant des établissements d'enseignement et de recherche français ou étrangers, des laboratoires publics ou privés. 


\title{
On constitutive inequalities in nonlinear theory of elastic shells
}

\author{
Victor A. Eremeyev ${ }^{1,2, *}$ and Leonid M. Zubov ${ }^{2, * *}$ \\ ${ }^{1}$ South Scientific Center of RASci, Milchakova stt., 8a, Rostov-on-Don, 344090, Russia \\ ${ }^{2}$ Rostov State University, Milchakova str., 8a, Rostov-on-Don, 344090, Russia
}

\section{In memory of Pavel Zhilin, our friend and teacher.}

\begin{abstract}
Constitutive inequalities in general static and dynamic theory of elastic shells undergoing finite deformation are discussed. Constitutive inequalities are well known in continuum mechanics. They express physical or mathematical restrictions for constitutive equations of 3D elastic materials. In this paper we discuss the analogs of the strong ellipticity, Hadamard and Coleman-Noll (GCN-condition) inequalities for nonlinear elastic shells. It is shown that the GCN-condition implies the strong elipticity for shell theory whereas the strong ellipticity is equivalent to the existence conditions of acceleration waves in shell.
\end{abstract}

\section{Introduction}

In nonlinear elasticity there are well known so-called constitutive restrictions. They are the strong ellipticity condition, Hadamard inequality, GCN-condition, and some others [1,2]. Each of them play some role in nonlinear elasticity. They express mathematically precise and physically intuitive restrictions for constitutive equations of elastic bodies.

The aim of this paper is to formulate similar constitutive inequalities in general nonlinear theory of micropolar or Cosserat's type shells. Here we formulate Coleman-Noll inequality, a strong ellipticity condition of equilibrium equations and Hadamard inequality. The inequalities represent possible restrictions of constitutive equations of elastic shells under finite deformation. We prove that Coleman-Noll inequality implies strong ellipticity of shell equilibrium equations.

Since the pioneering work by Cosserat brothers [3], two-dimensional micropolar bodies, i.e. plates and shells, were investigated, for example, in Altenbach and Zhilin [4, 5], Antman [6], Eremeyev [7], Eremeyev and Zubov [8], Shkutin [9], Zhilin $[10,11]$ and Zubov [12]. General nonlinear mechanics of shells with independent translation and rotation fields was developed by Chróścielewski et al. [13], Eremeyev and Pietraszkiewicz [17], Libai and Simmonds [14, 15], Sansour and Bednarczyk [18] and Pietraszkiewicz [16] as well as in many other papers, see, for example the references in [13]. Kinematic structure of this shell theory is identical to the classical version of the Cosserat surface. Mechanics of linear defects in micropolar shell is developed by Zubov [12]. The theory of material symmetry groups is developed by Eremeyev and Pietraszkiewicz [17] where the general constitutive equations of nonlinear elastic shell is considered.

First we will review basic relations of the nonlinear shell theory in the Sect. 2. Then we formulate the differential form of Coleman-Noll condition (an analog to the GCN-condition) (17) in the Sect. 3. In 3D nonlinear elasticity the so-called constitutive restrictions or constitutive inequalities are considered in [1,2]. From the physical point of view these restrictions express the our ideas on the physically reasonable behavior of materials. The GCN condition proposed by Coleman and Noll asserts "that the transformation from deformation gradient to first Piola-Kirchhoff stress tensor shall be monotone with respect to pairs of deformations differing from one another by a pure stretch" (see [1], pp. 162-163). Then we deduce the linearized equilibrium equations and formulate the strong ellipticity condition (19) and Hadamard inequality in the Sect. 4. We mention that Coleman-Noll condition is more general and it implies the strong ellipticity of the equilibrium shell equations. In Sect. 5 we also show that the strong ellipticity condition is equivalent to the conditions of the existence of accelerations waves in the shell.

\footnotetext{
* Corresponding author, e-mail: eremeyev@math.rsu. ru, Phone: +78632975282 , Fax: +78632975231

**e-mail: zubov@math.rsu.ru, Phone:+78632975108
} 


\section{Basic relations}

In this paper we use the so-called direct approach to the formulation of the basic equations of shell theory. The advantage of the latter approach was discussed in many papers, see for example [19]. Within framework of the direct approach, an elastic micropolar shell is a two-dimensional analogue of the Cosserat continuum, i.e. a micropolar shell is a material surface each particle of which has six degrees of freedom of the rigid body. Further we will use the notations $[7,8,12]$.

Let $\sigma$ be a base surface of the micropolar shell in a reference configuration (for example, in an undeformed state), $q^{\alpha}$ $(\alpha=1,2)$ be Gaussian coordinates on $\sigma$, and $\boldsymbol{r}\left(q^{1}, q^{2}\right)$ be a position vector of $\sigma$. In the actual (deformed) configuration the surface is denoted by $\Sigma$, and the position of its particles is given by the vector $\boldsymbol{R}\left(q^{1}, q^{2}\right)$.

Orientation of the particles is described by the so-called microrotation tensor (or turn-tensor) $\mathbf{H}\left(q^{1}, q^{2}\right)$, which is the proper orthogonal tensor. If we introduce three orthonormal vectors $\boldsymbol{d}_{k}(k=1,2,3)$, which describe the particle orientation in a reference configuration, and three orthonormal vectors $\boldsymbol{D}_{k}$, which determine the same particle orientation in an actual configuration, then the tensor $\mathbf{H}$ is given by $\mathbf{H}=\boldsymbol{d}_{k} \otimes \boldsymbol{D}_{k}$.

Thus, the micropolar shell is described by two kinematically independent fields

$$
\boldsymbol{R}=\boldsymbol{R}\left(q^{\alpha}\right), \quad \mathbf{H}=\mathbf{H}\left(q^{\alpha}\right) .
$$

For the micropolar shell made of an elastic material there should exist a strain energy density $W$. By using the principle of local action $[1,2]$ the constitutive equation for the function $W$ is given by the formula $[7,8]$

$$
W=W\left(\boldsymbol{R}, \nabla_{0} \boldsymbol{R}, \mathbf{H}, \nabla_{0} \mathbf{H}\right),
$$

where

$$
\nabla_{0} \Psi \triangleq \boldsymbol{r}^{\alpha} \otimes \frac{\partial \boldsymbol{\Psi}}{\partial q^{\alpha}}, \quad(\alpha, \beta=1,2) \quad \boldsymbol{r}^{\alpha} \cdot \boldsymbol{r}_{\beta}=\delta_{\beta}^{\alpha}, \quad \boldsymbol{r}^{\alpha} \cdot \boldsymbol{n}=0, \quad \boldsymbol{r}_{\beta}=\frac{\partial \boldsymbol{r}}{\partial q^{\beta}} .
$$

Here the vectors $\boldsymbol{r}_{\beta}$ and $\boldsymbol{r}^{\alpha}$ denote the natural and reciprocal bases on $\sigma, \boldsymbol{n}$ is the unit normal to $\sigma, \delta_{\beta}^{\alpha}$ is the Kronecker symbol, $\nabla_{0}$ is the surface nabla operator on $\sigma$, and $\boldsymbol{\Psi}$ is an arbitrary differentiable tensor field given on $\sigma$.

From the principle of material frame-indifference we can find that, in fact, $W$ depends only on two Cosserat type strain measures $\mathbf{Y}$ and $\mathbf{L}$

$$
\begin{aligned}
& W=W(\mathbf{Y}, \mathbf{L}), \\
& \mathbf{Y}=\left(\nabla_{0} \boldsymbol{R}\right) \cdot \mathbf{H}^{T}, \quad \mathbf{L}=\frac{1}{2} \boldsymbol{r}^{\alpha} \otimes\left(\frac{\partial \mathbf{H}}{\partial q^{\alpha}} \cdot \mathbf{H}^{T}\right)_{\times},
\end{aligned}
$$

where $\mathbf{T}_{\times}$is the vectorial invariant of a second rank tensor $\mathbf{T}$ defined by $\mathbf{T}_{\times}=\left(T^{m n} \boldsymbol{R}_{m} \otimes \boldsymbol{R}_{n}\right)_{\times}=T^{m n} \boldsymbol{R}_{m} \times \boldsymbol{R}_{n}$ for any base $\boldsymbol{R}_{m}$ [20], $\times$ is the vector product.

The Lagrangian equilibrium equations of the micropolar shell can be derived from the principle of virtual work

$$
\delta \iint_{\sigma} W d \sigma=\delta^{\prime} A
$$

where

$$
\delta^{\prime} A=\iint_{\sigma}(\boldsymbol{f} \cdot \delta \boldsymbol{R}+\boldsymbol{\ell} \cdot \boldsymbol{\psi}) d \sigma+\int_{\omega_{2}} \boldsymbol{\varphi} \cdot \delta \boldsymbol{R} d s+\int_{\omega_{4}} \boldsymbol{\gamma} \cdot \boldsymbol{\psi} d s, \quad \mathbf{I} \times \boldsymbol{\psi}=-\mathbf{H}^{T} \cdot \delta \mathbf{H} .
$$

In the relation (2), $\delta$ is the symbol of variation, $\boldsymbol{\psi}$ is the virtual rotation vector, $\boldsymbol{f}$ is the surface force density distributed on $\sigma, \ell$ is the surface couple density distributed on $\sigma, \varphi$, and $\gamma$ are linear densities of forces and couples distributed along corresponding parts of the shell boundary $\omega$, and $\mathbf{I}$ is the $3 \mathrm{D}$ unit tensor, respectively.

Thus, the Lagrangian shell equations take the form

$$
\begin{aligned}
& \nabla_{0} \cdot \mathbf{D}+\boldsymbol{f}=\mathbf{0}, \quad \nabla_{0} \cdot \mathbf{G}+\left[\left(\nabla_{0} \boldsymbol{R}\right)^{T} \cdot \mathbf{D}\right]_{\times}+\boldsymbol{\ell}=\mathbf{0}, \\
& \mathbf{D}=\mathbf{P}_{1} \cdot \mathbf{H}, \quad \mathbf{G}=\mathbf{P}_{2} \cdot \mathbf{H}, \quad \mathbf{P}_{1}=\frac{\partial W}{\partial \mathbf{Y}}, \quad \mathbf{P}_{2}=\frac{\partial W}{\partial \mathbf{L}},
\end{aligned}
$$




$$
\begin{aligned}
\omega_{1} & : \boldsymbol{R}=\boldsymbol{\rho}(s), \\
\omega_{2} & : \boldsymbol{\nu} \cdot \mathbf{D}=\boldsymbol{\varphi}(s), \\
\omega_{3} & : \mathbf{H}=\mathbf{h}(s), \mathbf{h} \cdot \mathbf{h}^{T}=\mathbf{I}, \\
\omega_{4} & : \boldsymbol{\nu} \cdot \mathbf{G}=\gamma(s) .
\end{aligned}
$$

Here $\boldsymbol{\rho}(s), \boldsymbol{h}(s)$ are given vector functions, and $\boldsymbol{\nu}$ is the external unit normal to the boundary curve $\omega(\boldsymbol{\nu} \cdot \boldsymbol{n}=0)$. The relations (3) are the equilibrium equations for the linear momentum and angular momentum of any shell part. The tensors $\mathbf{D}$ and $\mathbf{G}$ are the surface stress and couple stress tensors of the Piola type, and the corresponding stress measures $\mathbf{P}_{1}$ and $\mathbf{P}_{2}$ in the Eqs. (3) are the Kirchoff type tensors, respectively. The boundary $\omega$ of $\sigma$ is divided into two parts $\omega=\omega_{1} \cup \omega_{2}=\omega_{3} \cup \omega_{4}$. The following relations hold:

$$
\boldsymbol{n} \cdot \mathrm{D}=\boldsymbol{n} \cdot \mathrm{G}=\boldsymbol{n} \cdot \mathrm{P}_{1}=\boldsymbol{n} \cdot \mathrm{P}_{2}=\mathbf{0}
$$

The equilibrium equations (3) may be transformed to the Eulerian form

$$
\nabla \cdot \mathbf{T}+J^{-1} \boldsymbol{f}=\mathbf{0}, \quad \nabla \cdot \mathbf{M}+\mathbf{T}_{\times}+J^{-1} \ell=\mathbf{0},
$$

where

$$
\begin{aligned}
& \nabla \cdot \Psi \triangleq \boldsymbol{R}^{\alpha} \cdot \frac{\partial \boldsymbol{\Psi}}{\partial q^{\alpha}}, \quad \boldsymbol{R}^{\alpha} \cdot \boldsymbol{R}_{\beta}=\delta_{\beta}^{\alpha}, \quad \boldsymbol{R}^{\alpha} \cdot \mathbf{N}=0, \quad \boldsymbol{R}_{\beta}=\frac{\partial \boldsymbol{R}}{\partial q^{\beta}} \\
& \mathbf{T}=J^{-1} \mathbf{F}^{T} \cdot \mathbf{D}, \quad \mathbf{M}=J^{-1} \mathbf{F}^{T} \cdot \mathbf{G}, \\
& \mathbf{F}=\nabla_{0} \boldsymbol{R}, \quad J=\sqrt{\frac{1}{2}\left[\operatorname{tr}^{2}\left(\mathbf{F} \cdot \mathbf{F}^{T}\right)-\operatorname{tr}\left(\mathbf{F} \cdot \mathbf{F}^{T}\right)^{2}\right]} .
\end{aligned}
$$

Here $\mathbf{T}$ and $\mathbf{M}$ are the Cauchy type surface stress and couple stress tensors, $\nabla$ is the surface nabla operator on $\Sigma$ associated with $\nabla_{0}$ by formula $\nabla_{0}=\mathbf{F} \cdot \nabla$.

The equations of motion of the micropolar shell are given by the relations (see Altenbach and Zhilin [4], Libai and Simmonds [14,15], Zhilin [11])

$$
\begin{aligned}
& \nabla_{0} \cdot \mathbf{D}+\boldsymbol{f}=\rho \frac{\mathrm{d} \boldsymbol{K}_{1}}{\mathrm{~d} t}, \quad \nabla_{0} \cdot \mathbf{G}+\left[\mathbf{F}^{T} \cdot \mathbf{D}\right]_{\times}+\boldsymbol{m}=\rho\left(\frac{\mathrm{d} \boldsymbol{K}_{2}}{\mathrm{~d} t}+\boldsymbol{v} \times \boldsymbol{\Theta}_{1}^{T} \cdot \boldsymbol{\omega}\right) \\
& K(\boldsymbol{v}, \boldsymbol{\omega})=\frac{1}{2} \boldsymbol{v} \cdot \boldsymbol{v}+\boldsymbol{\omega} \cdot \boldsymbol{\Theta}_{1} \cdot \boldsymbol{v}+\frac{1}{2} \boldsymbol{\omega} \cdot \boldsymbol{\Theta}_{2} \cdot \boldsymbol{\omega}, \quad \boldsymbol{K}_{1} \triangleq \frac{\partial K}{\partial \boldsymbol{v}}=\boldsymbol{v}+\boldsymbol{\Theta}_{1}^{T} \cdot \boldsymbol{\omega}, \quad \boldsymbol{K}_{2} \triangleq \frac{\partial K}{\partial \boldsymbol{\omega}}=\boldsymbol{\Theta}_{1} \cdot \boldsymbol{v}+\boldsymbol{\Theta}_{2} \cdot \boldsymbol{\omega},
\end{aligned}
$$

where $\boldsymbol{v}=\frac{d \boldsymbol{R}}{d t}$ and $\boldsymbol{\omega}=\frac{1}{2}\left(\mathbf{H}^{T} \cdot \frac{d \mathbf{H}}{d t}\right)_{\times}$are the linear and angular velocities, respectively, $\rho$ is the surface mass density in the reference configuration, $\rho K$ is the surface density of kinetic energy, and $\rho \boldsymbol{\Theta}_{1}, \rho \boldsymbol{\Theta}_{2}$ are the rotary inertia tensors $\left(\Theta_{2}^{T}=\Theta_{2}\right)$.

For the dynamic problem (7), the initial conditions are given by

$$
\left.\boldsymbol{R}\right|_{t=0}=\boldsymbol{R}^{\circ},\left.\quad \boldsymbol{v}\right|_{t=0}=\boldsymbol{v}^{\circ},\left.\quad \mathbf{H}\right|_{t=0}=\mathbf{H}^{\circ},\left.\quad \boldsymbol{\omega}\right|_{t=0}=\boldsymbol{\omega}^{\circ},
$$

where $\boldsymbol{R}^{\circ}, \boldsymbol{v}^{\circ}, \mathbf{H}^{\circ}, \boldsymbol{\omega}^{\circ}$ are given initial values.

As an example, the following quadratic form may be used as the constitutive equation of the isotropic shell $[8,17]$ :

$$
\begin{aligned}
2 W= & \alpha_{1} \operatorname{tr}^{2} \mathbf{Y}_{\|}+\alpha_{2} \operatorname{tr} \mathbf{Y}_{\|}^{2}+\alpha_{3} \operatorname{tr}\left(\mathbf{Y}_{\|} \cdot \mathbf{Y}_{\|}^{T}\right)+\alpha_{4} \boldsymbol{n} \cdot \mathbf{Y}^{T} \cdot \mathbf{Y} \cdot \boldsymbol{n} \\
& +\beta_{1} \operatorname{tr}^{2} \mathbf{L}_{\|}+\beta_{2} \operatorname{tr} \mathbf{L}_{\|}^{2}+\beta_{3} \operatorname{tr}\left(\mathbf{L}_{\|} \cdot \mathbf{L}_{\|}^{T}\right)+\beta_{4} \boldsymbol{n} \cdot \mathbf{L}^{T} \cdot \mathbf{L} \cdot \boldsymbol{n}
\end{aligned}
$$

where $\alpha_{i}, \beta_{i}$ are the material constants $(i=1, \ldots, 4), \mathbf{Y}_{\|} \triangleq(\mathbf{Y}-\mathbf{I}) \cdot \mathbf{g}, \mathbf{L}_{\|} \triangleq \mathbf{L} \cdot \mathbf{g}, \mathbf{g} \triangleq \mathbf{I}-\boldsymbol{n} \otimes \boldsymbol{n}$. 


\section{Coleman-Noll inequality for elastic shells}

Let us suppose that a certain equilibrium state of a nonlinear elastic shell of the Cosserat type under the action of a given load is known. Further we will call it as initial or basic stressed state. This state could be defined by the vector field $\boldsymbol{R}\left(q^{\alpha}\right)$ and tensor field $\mathbf{H}\left(q^{\alpha}\right)$. Along with the basic stressed state we consider some perturbed equilibrium state which differs from the basic one. The linear parts of increments of different quantities that characterize the perturbed equilibrium we will denote using a dot above, for example

$$
\mathbf{D}^{\cdot}=\left.\frac{\mathrm{d}}{\mathrm{d} \tau} \mathbf{D}\left[\nabla_{0}\left(\boldsymbol{R}+\tau \boldsymbol{u}, \mathbf{H}-\tau \mathbf{H} \times \boldsymbol{\theta}, \nabla_{0}(\mathbf{H}-\tau \mathbf{H} \times \boldsymbol{\theta})\right)\right]\right|_{\tau=0} .
$$

Here $\boldsymbol{u}$ is the vector of additional infinitesimal displacement, while $\boldsymbol{\theta}$ is the vector of additional infinitesimal rotation characterizing the small rotation with respect to initial stressed state. The following relations hold

$$
\begin{aligned}
& \boldsymbol{R}=\boldsymbol{u}, \quad \mathbf{H}^{\cdot}=-\mathbf{H} \times \boldsymbol{\theta}, \quad \mathbf{Y} \cdot=\mathbf{F} \cdot \varepsilon \cdot \mathbf{H}^{T}, \quad \mathbf{L}=\mathbf{F} \cdot \varkappa \cdot \mathbf{H}^{T}, \\
& \varepsilon=\nabla \boldsymbol{u}+\mathbf{I} \times \boldsymbol{\theta}, \quad \varkappa=\nabla \boldsymbol{\theta},
\end{aligned}
$$

where $\varepsilon$ is the strain tensor and $\varkappa$ is the bending strain tensor usually used in the linear shell theory of the Cosserat type $[10,12]$.

Note that as a reference configuration may be chosen any stressed state of a shell. To avoid an awkward expressions and to simplify the calculations let us assume that the reference configuration coincides with the initial (basic) stressed state of the shell. This means that in the reference configuration $\mathbf{F}=\mathbf{Y}=\mathbf{I}-\boldsymbol{n} \otimes \boldsymbol{n}, \mathbf{H}=\mathbf{I}, \mathbf{L}=\mathbf{0}$. Under this choice of reference configuration and using (4), (6), (9)-(11) we obtain

$$
\mathbf{D}^{*}=\frac{\partial^{2} W}{\partial \mathbf{Y} \partial \mathbf{Y}} \circ \varepsilon+\frac{\partial^{2} W}{\partial \mathbf{Y} \partial \mathbf{L}} \circ \varkappa-\mathbf{T} \times \boldsymbol{\theta}, \quad \mathbf{G}^{\cdot}=\frac{\partial^{2} W}{\partial \mathbf{L} \partial \mathbf{Y}} \circ \varepsilon+\frac{\partial^{2} W}{\partial \mathbf{L} \partial \mathbf{L}} \circ \varkappa-\mathbf{M} \times \boldsymbol{\theta} .
$$

Here and below we use the operation of a scalar product of tensors given by formulae

$$
\begin{aligned}
& \left(C^{k s t p} \boldsymbol{R}_{k} \otimes \boldsymbol{R}_{s} \otimes \boldsymbol{R}_{t} \otimes \boldsymbol{R}_{p}\right) \circ\left(U_{m n} \boldsymbol{R}^{m} \otimes \boldsymbol{R}^{n}\right) \triangleq C^{k s m n} U_{m n} \boldsymbol{R}_{k} \otimes \boldsymbol{R}_{s}, \\
& \left(V_{i j} \boldsymbol{R}^{i} \otimes \boldsymbol{R}^{j}\right) \circ\left(C^{k s t p} \boldsymbol{R}_{k} \otimes \boldsymbol{R}_{s} \otimes \boldsymbol{R}_{t} \otimes \boldsymbol{R}_{p}\right) \circ\left(U_{m n} \boldsymbol{R}^{m} \otimes \boldsymbol{R}^{n}\right) \triangleq C^{i j m n} V_{i j} U_{m n} .
\end{aligned}
$$

Suppose that the external couples in the initial and perturbed stressed states of a shell vanish $\ell=\gamma=0$, while the external forces are "dead". Then the elastic energy of a shell is given by the relation

$$
\Pi=\iint_{\sigma} W \mathrm{~d} \sigma-\iint_{\sigma} \boldsymbol{f} \cdot(\boldsymbol{R}-\boldsymbol{r}) \mathrm{d} \sigma-\int_{\omega_{2}} \boldsymbol{\varphi} \cdot(\boldsymbol{R}-\boldsymbol{r}) \mathrm{d} s .
$$

Let us consider the energy increment in the perturbed equilibrium state with respect to the energy in the initial state taking into account terms of order one and two

$$
\Pi-\Pi_{0}=\tau\left(\frac{\mathrm{d} \Pi}{\mathrm{d} \tau}\right)_{\tau=0}+\frac{1}{2} \tau^{2}\left(\frac{\mathrm{d}^{2} \Pi}{\mathrm{d} \tau^{2}}\right)_{\tau=0}+\ldots
$$

According to the constitutive relations of an elastic shell (4) and formulae (10), (11) we obtain

$$
\frac{\mathrm{d} \Pi}{\mathrm{d} \tau}=\iint_{\sigma}\left[\operatorname{tr}\left(\mathbf{D}^{T} \cdot \nabla_{0} \boldsymbol{u}\right)+\operatorname{tr}\left(\mathbf{D}^{T} \cdot \mathbf{F} \times \boldsymbol{\theta}\right)+\operatorname{tr}\left(\mathbf{G}^{T} \cdot \nabla_{0} \boldsymbol{\theta}\right)\right] \mathrm{d} \sigma-\iint_{\sigma} \boldsymbol{f} \cdot \boldsymbol{u} \mathrm{d} \sigma-\int_{\omega_{2}} \boldsymbol{\varphi} \cdot \boldsymbol{u} \mathrm{d} s .
$$

Differentiating (13) with respect to parameter $\tau$ and taking into account that the reference configuration coincides with the basic stressed state and equations (10) we obtain

$$
\left.\frac{\mathrm{d}^{2} \Pi}{\mathrm{d} \tau^{2}}\right|_{\tau=0}=\iint_{\Sigma}\left[\operatorname{tr}\left(\mathbf{D}^{\cdot T} \cdot \nabla \boldsymbol{u}\right)+\operatorname{tr}\left(\mathbf{D}^{\cdot T} \times \boldsymbol{\theta}\right)+\operatorname{tr}\left(\mathbf{T}^{T} \cdot(\nabla \boldsymbol{u}) \times \boldsymbol{\theta}\right)+\operatorname{tr}\left(\mathbf{G}^{\cdot T} \cdot \varkappa\right)\right] \mathrm{d} \Sigma .
$$


From the equilibrium condition of the basic state and in virtue of (3) and (5) it follows that the first variation of the energy $\left.\frac{\mathrm{d} \Pi}{\mathrm{d} \tau}\right|_{\tau=0}$ vanish, and the second variation due to (11) and (12) could be represented as

$$
\begin{aligned}
& \left.\frac{\mathrm{d}^{2} \Pi}{\mathrm{d} \tau^{2}}\right|_{\tau=0}=2 \iint_{\Sigma} w \mathrm{~d} \Sigma, \quad w=w^{\prime}+w^{\prime \prime}, \\
& w^{\prime}=\frac{1}{2} \varepsilon \circ \frac{\partial^{2} W}{\partial \mathbf{Y} \partial \mathbf{Y}} \circ \varepsilon+\varepsilon \circ \frac{\partial^{2} W}{\partial \mathbf{Y} \partial \mathbf{L}} \circ \varkappa+\frac{1}{2} \varkappa \circ \frac{\partial^{2} W}{\partial \mathbf{L} \partial \mathbf{L}} \circ \varkappa, \\
& w^{\prime \prime}=\operatorname{tr}\left(\boldsymbol{\theta} \times \mathbf{T}^{T} \cdot \boldsymbol{\varepsilon}\right)-\frac{1}{2} \operatorname{tr}\left(\boldsymbol{\theta} \times \mathbf{T}^{T} \times \boldsymbol{\theta}\right)+\frac{1}{2} \operatorname{tr}\left(\boldsymbol{\theta} \times \mathbf{M}^{T} \cdot \varkappa\right) .
\end{aligned}
$$

The quantity $w$ describes the increment of the elastic energy of initially prestressed shell under additional infinitesimal deformations. According to Eqs. (14) and (15) this energy may be decomposed into two parts: the energy of a pure strain $w^{\prime}$ and the energy of rotations $w^{\prime \prime}$. The coefficients in the quadratic form $w^{\prime \prime}$ are expressed in terms of the stress and couple tensors of the initial stressed state and do not depend on material properties of the shell. If the basic stressed state of the shell is natural ( $\mathbf{T}=\mathbf{M}=\mathbf{0}$ ), then $w=w^{\prime}$ and the energy density reduces to a quadratic form of tensors $\varepsilon$ and $\varkappa$. If the the decomposition (14) and the formulae (15) are compared with the similar results on increment of 3D strain energy density [21] of micropolar body one can easily note that they coincide up to notation.

The one of well-known in the nonlinear elasticity $[1,2]$ constitutive inequalities is Coleman-Noll inequality. The differential form of Coleman-Noll inequality (so-called GCN-condition) expresses a property of positivity of the elastic energy density under of any infinitesimal non-zero pure strains and bending strains for any arbitrary reference configuration. It is necessary to mention that this inequality does not restrict on the constitutive equations with regard to any rotations.

Taking into account the energy decomposition (14) we obtain an analog of Coleman-Noll inequality for elastic shells of the Cosserat type

$$
w^{\prime}(\varepsilon, \varkappa)>0 \quad \forall \varepsilon \neq \mathbf{0}, \quad \varkappa \neq \mathbf{0} .
$$

Using formulae (15) inequality (16) can be written in the equivalent form

$$
\left.\frac{\mathrm{d}^{2}}{\mathrm{~d} \tau^{2}} W(\mathbf{Y}+\tau \varepsilon, \mathbf{L}+\tau \varkappa)\right|_{\tau=0}>0 \quad \forall \varepsilon \neq \mathbf{0}, \quad \varkappa \neq \mathbf{0} .
$$

Condition (17) satisfies the principle of material frame-indifference and could serve as a constitutive inequality for elastic shells.

\section{Strong ellipticity and Hadamard inequality}

The famous constitutive inequalities in the nonlinear elasticity are the strong ellipticity condition and its weak form known as Hadamard inequality. Following the theory of the system of partial differential equations (PDE) $[22,23]$ in this section we formulate the strong ellipticity condition of the equilibrium equations (3). In the case of dead loading the linearized equilibrium equations have the form

$$
\nabla_{0} \cdot \mathbf{D} \cdot=\mathbf{0}, \quad \nabla_{0} \cdot \mathbf{G} \cdot\left[\mathbf{F}^{T} \cdot \mathbf{D} \cdot\left(\nabla_{0} \boldsymbol{u}\right)^{T} \cdot \mathbf{D}\right]_{\times}=\mathbf{0},
$$

where $\mathbf{D}^{\bullet}$ and $\mathbf{G}^{\cdot}$ are given by formulae similar to (9). Eqs. (18) are the system of linear PDE of second order with respect to $\boldsymbol{u}$ and $\boldsymbol{\theta}$. If we keep in Eqs. (18) the differential operators of second order we obtain the relations

$$
\begin{aligned}
& \nabla_{0} \cdot\left\{\left[\frac{\partial^{2} W}{\partial \mathbf{Y} \partial \mathbf{Y}} \circ\left(\left(\nabla_{0} \boldsymbol{u}\right) \cdot \mathbf{H}^{T}\right)+\frac{\partial^{2} W}{\partial \mathbf{Y} \partial \mathbf{L}} \circ\left(\left(\nabla_{0} \boldsymbol{\theta}\right) \cdot \mathbf{H}^{T}\right)\right] \cdot \mathbf{H}\right\}, \\
& \nabla_{0} \cdot\left\{\left[\frac{\partial^{2} W}{\partial \mathbf{L} \partial \mathbf{Y}} \circ\left(\left(\nabla_{0} \boldsymbol{u}\right) \cdot \mathbf{H}^{T}\right)+\frac{\partial^{2} W}{\partial \mathbf{L} \partial \mathbf{L}} \circ\left(\left(\nabla_{0} \boldsymbol{\theta}\right) \cdot \mathbf{H}^{T}\right)\right] \cdot \mathbf{H}\right\},
\end{aligned}
$$

which allow one to construct the condition of strong ellipticity of the system (18). Using formal procedure [22, 23] we replace differential operator $\nabla_{0}$ to the unit vector $\boldsymbol{\nu}$ tangential to the surface $\sigma$, while the vector fields $\boldsymbol{u}$ and $\boldsymbol{\theta}$ to the vectors $\boldsymbol{a}$ and $\boldsymbol{b}$, respectively. Thus, we have the algebraic expressions

$$
\boldsymbol{\nu} \cdot\left\{\left[\frac{\partial^{2} W}{\partial \mathbf{Y} \partial \mathbf{Y}} \circ\left(\boldsymbol{\nu} \otimes \boldsymbol{a} \cdot \mathbf{H}^{T}\right)+\frac{\partial^{2} W}{\partial \mathbf{Y} \partial \mathbf{L}} \circ\left(\boldsymbol{\nu} \otimes \boldsymbol{b} \cdot \mathbf{H}^{T}\right)\right] \cdot \mathbf{H}\right\},
$$




$$
\boldsymbol{\nu} \cdot\left\{\left[\frac{\partial^{2} W}{\partial \mathbf{L} \partial \mathbf{Y}} \circ\left(\boldsymbol{\nu} \otimes \boldsymbol{a} \cdot \mathbf{H}^{T}\right)+\frac{\partial^{2} W}{\partial \mathbf{L} \partial \mathbf{L}} \circ\left(\boldsymbol{\nu} \otimes \boldsymbol{b} \cdot \mathbf{H}^{T}\right)\right] \cdot \mathbf{H}\right\}
$$

Let us multiply the first equation to the vector $\boldsymbol{a}$ while the second one to the vector $\boldsymbol{b}$ and add these expressions. Then we obtain the strong ellipticity condition of the equations (18) in the following form

$$
\begin{aligned}
\boldsymbol{\nu} \cdot\left\{\left[\frac{\partial^{2} W}{\partial \mathbf{Y} \partial \mathbf{Y}} \circ\left(\boldsymbol{\nu} \otimes \boldsymbol{a} \cdot \mathbf{H}^{T}\right)+\frac{\partial^{2} W}{\partial \mathbf{Y} \partial \mathbf{L}} \circ\left(\boldsymbol{\nu} \otimes \boldsymbol{b} \cdot \mathbf{H}^{T}\right)\right] \cdot \mathbf{H}\right\} \cdot \boldsymbol{a} \\
\quad+\boldsymbol{\nu} \cdot\left\{\left[\frac{\partial^{2} W}{\partial \mathbf{L} \partial \mathbf{Y}} \circ\left(\boldsymbol{\nu} \otimes \boldsymbol{a} \cdot \mathbf{H}^{T}\right)+\frac{\partial^{2} W}{\partial \mathbf{L} \partial \mathbf{L}} \circ\left(\boldsymbol{\nu} \otimes \boldsymbol{b} \cdot \mathbf{H}^{T}\right)\right] \cdot \mathbf{H}\right\} \cdot \boldsymbol{b}>0, \quad \forall \boldsymbol{a}, \boldsymbol{b} \neq \mathbf{0} .
\end{aligned}
$$

Using operation $\circ$ we can transform the latter equations as follows

$$
\begin{aligned}
& \left(\boldsymbol{\nu} \otimes \boldsymbol{a} \cdot \mathbf{H}^{T}\right) \circ \frac{\partial^{2} W}{\partial \mathbf{Y} \partial \mathbf{Y}} \circ\left(\boldsymbol{\nu} \otimes \boldsymbol{a} \cdot \mathbf{H}^{T}\right)+2\left(\boldsymbol{\nu} \otimes \boldsymbol{a} \cdot \mathbf{H}^{T}\right) \circ \frac{\partial^{2} W}{\partial \mathbf{Y} \partial \mathbf{L}} \circ\left(\boldsymbol{\nu} \otimes \boldsymbol{b} \cdot \mathbf{H}^{T}\right) \\
& +\left(\boldsymbol{\nu} \otimes \boldsymbol{b} \cdot \mathbf{H}^{T}\right) \circ \frac{\partial^{2} W}{\partial \mathbf{L} \partial \mathbf{L}} \circ\left(\boldsymbol{\nu} \otimes \boldsymbol{b} \cdot \mathbf{H}^{T}\right)>0, \quad \forall \boldsymbol{a}, \boldsymbol{b} \neq \mathbf{0} .
\end{aligned}
$$

This inequality can be written in a more compact form using the matrix notation

$$
\boldsymbol{\xi} \cdot \mathbb{A}(\boldsymbol{\nu}) \cdot \boldsymbol{\xi}>0, \quad \forall \boldsymbol{\nu} \in \mathcal{T}_{x} \sigma, \quad \boldsymbol{\nu} \neq \mathbf{0}, \quad \forall \boldsymbol{\xi} \in \mathbb{R}^{6}, \quad \boldsymbol{\xi} \neq \mathbf{0},
$$

where $\boldsymbol{\xi}=\left(\boldsymbol{a}^{\prime}, \boldsymbol{b}^{\prime}\right) \in \mathbb{R}^{6}, \boldsymbol{a}^{\prime}=\boldsymbol{a} \cdot \mathbf{H}^{T}, \boldsymbol{b}^{\prime}=\boldsymbol{b} \cdot \mathbf{H}^{T}$, and the matrix $\mathbb{A}(\boldsymbol{\nu})$ is given by

$$
\mathbb{A}(\boldsymbol{\nu}) \triangleq\left[\begin{array}{cc}
\frac{\partial^{2} W}{\partial \mathbf{Y} \partial \mathbf{Y}}\{\boldsymbol{\nu}\} & \frac{\partial^{2} W}{\partial \mathbf{Y} \partial \mathbf{L}}\{\boldsymbol{\nu}\} \\
\frac{\partial^{2} W}{\partial \mathbf{L} \partial \mathbf{Y}}\{\boldsymbol{\nu}\} & \frac{\partial^{2} W}{\partial \mathbf{L} \partial \mathbf{L}}\{\boldsymbol{\nu}\}
\end{array}\right],
$$

where for any fourth rank tensor $\mathbf{K}$ and any vector $\boldsymbol{\nu}: \mathbf{K}\{\boldsymbol{\nu}\} \triangleq K_{k l m n} \nu_{k} \nu_{m} \boldsymbol{i}_{l} \otimes \boldsymbol{i}_{n}$.

The inequality (19) is the strong ellipticity condition of the equilibrium equations (3) of an elastic shells. A weak form of the inequality (19) is an analogue of Hadamard inequality for the shell. These inequalities is an example of possible restrictions of the constitutive equations of elastic shells under finite deformations. As in the case of simple materials, a break in inequality (19) means the possibility of existing nonsmooth solutions of the equilibrium equations (3).

As an example we consider the constitutive equations of an isotropic micropolar shell (8). In this case the inequality (19) reduces to the system of inequalities for elastic constants

$$
\alpha_{3}>0, \quad \alpha_{1}+\alpha_{2}+\alpha_{3}>0, \quad \alpha_{4}>0, \quad \beta_{3}>0, \quad \beta_{1}+\beta_{2}+\beta_{3}>0, \quad \beta_{4}>0 .
$$

The strong ellipticity condition may we written in the equivalent form

$$
\left.\frac{\mathrm{d}^{2}}{\mathrm{~d} \tau^{2}} W\left(\mathbf{Y}+\tau \boldsymbol{\nu} \otimes \boldsymbol{a}^{\prime}, \mathbf{L}+\tau \boldsymbol{\nu} \otimes \boldsymbol{b}^{\prime}\right)\right|_{\tau=0}>0 \quad \forall \boldsymbol{\nu}, \boldsymbol{a}^{\prime}, \boldsymbol{b}^{\prime} \neq \mathbf{0} .
$$

Comparing the condition of strong ellipticity (20) and the Coleman-Noll inequality (17) one can see that the latter implies the former. Indeed, the inequality (17) holds for any tensors $\varepsilon$ and $\varkappa$. Note, that the tensors $\varepsilon$ and $\varkappa$ may by nonsymmetric, in general. If we substitute in the inequality (17) the relations $\varepsilon=\boldsymbol{\nu} \otimes \boldsymbol{a}^{\prime}$ and $\varkappa=\boldsymbol{\nu} \otimes \boldsymbol{b}^{\prime}$ then we immediately obtain the inequality (20). Thus, the strong ellipticity condition is the special case of Coleman-Noll inequality. This is the essential distinction between the shell theory of the Cosserat type and the theory of simple elastic materials $[1,2]$ where these two properties completely independent of each other in the sense that neither of them implies the other one. For 3D elastic micropolar media the strong ellipticity and Hadamard conditions are formulated in [21].

\section{Strong ellipticity condition and acceleration waves}

Using approach [24], we show that the inequality (19) coincides with the conditions for propagation of acceleration waves in a shell. We consider a motion that may be accompanied by a jump in the continuity of kinematic and dynamic quantities on a certain smooth curve $C(t)$, which is called singular. We assume that the limiting values of these quantities exist on $C$ and that they are generally different on the opposite sides of $C$. The jump of a any quantity $\boldsymbol{\Psi}$ on $C$ is denoted as $\llbracket \Psi \rrbracket=\Psi^{+}-\Psi^{-}$. 
The acceleration wave (weak-discontinuity wave or second-order singular curve) is a moving singular curve $C$ on which the second derivatives (with respect to the spatial coordinates and time) of the radius-vector $\boldsymbol{R}$ and microrotation tensor $\mathbf{H}$ are discontinuous, while the quantities themselves and their first derivatives are continuous; i.e., the equalities

$$
\llbracket \mathbf{F} \rrbracket=\mathbf{0}, \quad \llbracket \nabla_{0} \mathbf{H} \rrbracket=\mathbf{0}, \quad \llbracket \boldsymbol{v} \rrbracket=\mathbf{0}, \quad \llbracket \boldsymbol{\omega} \rrbracket=\mathbf{0},
$$

are valid on $C$. According to Eqs. (1), the strain measure $\mathbf{Y}$ and the bending strain tensor $\mathbf{L}$ are continuous near $C$, and, in view of constitutive equations (4), jumps of the tensors $\mathbf{D}$ and $\mathbf{G}$ are absent. The application of the Maxwell theorem [2] to continuous fields of velocities $\boldsymbol{v}$ and $\boldsymbol{\omega}$, surface stress tensor $\mathbf{D}$, and the surface couple stress tensor $\mathbf{G}$ yields a system of equations that relate the jumps of their derivatives with respect to the spatial coordinates and time:

$$
\begin{aligned}
& \llbracket \frac{\mathrm{d} \boldsymbol{v}}{\mathrm{d} t} \rrbracket=-V \boldsymbol{a}, \quad \llbracket \nabla_{0} \boldsymbol{v} \rrbracket=\boldsymbol{\nu} \otimes \boldsymbol{a}, \quad \llbracket \frac{\mathrm{d} \boldsymbol{\omega}}{\mathrm{d} t} \rrbracket=-V \boldsymbol{b}, \quad \llbracket \nabla_{0} \boldsymbol{\omega} \rrbracket=\boldsymbol{\nu} \otimes \boldsymbol{b}, \\
& V \llbracket \nabla_{0} \cdot \mathbf{D} \rrbracket=-\boldsymbol{\nu} \cdot \llbracket \frac{\mathrm{d} \mathbf{D}}{\mathrm{d} t} \rrbracket, \quad V \llbracket \nabla_{0} \cdot \mathbf{G} \rrbracket=-\boldsymbol{\nu} \cdot \llbracket \frac{\mathrm{d} \mathbf{G}}{\mathrm{d} t} \rrbracket .
\end{aligned}
$$

Here, $\boldsymbol{a}$ and $\boldsymbol{b}$ are the vector amplitudes for the jumps of the linear and angular accelerations, $\boldsymbol{\nu}$ is the unit normal vector to $C$ such that $\boldsymbol{n} \cdot \boldsymbol{\nu}=0$, and $V$ is the velocity of the surface $C$ in the direction $\boldsymbol{\nu}$. If external forces and couples are continuous, the relations

$$
\llbracket \nabla_{0} \cdot \mathbf{D} \rrbracket=\rho \llbracket \frac{\boldsymbol{K}_{1}}{\mathrm{~d} t} \rrbracket, \quad \llbracket \nabla_{0} \cdot \mathbf{G} \rrbracket=\rho \gamma \llbracket \frac{\boldsymbol{K}_{2}}{\mathrm{~d} t} \rrbracket
$$

follow immediately from the equations of motion (7).

Differentiating constitutive equations (4) and using Eqs. (21) and (22), we express latter relations only in terms of the vector amplitudes $\boldsymbol{a}$ and $\boldsymbol{b}$ :

$$
\begin{aligned}
& \boldsymbol{\nu} \cdot \frac{\partial^{2} W}{\partial \mathbf{Y} \partial \mathbf{Y}} \circ\left(\boldsymbol{\nu} \otimes \boldsymbol{a} \cdot \mathbf{H}^{T}\right)+\boldsymbol{\nu} \cdot \frac{\partial^{2} W}{\partial \mathbf{Y} \partial \mathbf{L}} \circ\left(\boldsymbol{\nu} \otimes \boldsymbol{b} \cdot \mathbf{H}^{T}\right)=\rho V^{2}\left[\boldsymbol{a} \cdot \mathbf{H}^{T}+\left(\mathbf{H} \cdot \boldsymbol{\Theta}_{1}^{T} \cdot \mathbf{H}^{T}\right) \cdot\left(\boldsymbol{b} \cdot \mathbf{H}^{T}\right)\right], \\
& \boldsymbol{\nu} \cdot \frac{\partial^{2} W}{\partial \mathbf{L} \partial \mathbf{Y}} \circ\left(\boldsymbol{\nu} \otimes \boldsymbol{a} \cdot \mathbf{H}^{T}\right)+\boldsymbol{\nu} \cdot \frac{\partial^{2} W}{\partial \mathbf{L} \partial \mathbf{L}} \circ\left(\boldsymbol{\nu} \otimes \boldsymbol{b} \cdot \mathbf{H}^{T}\right)=\rho V^{2}\left[\left(\mathbf{H} \cdot \boldsymbol{\Theta}_{1} \cdot \mathbf{H}^{T}\right) \cdot\left(\boldsymbol{a} \cdot \mathbf{H}^{T}\right)\right. \\
& \left.+\left(\mathbf{H} \cdot \boldsymbol{\Theta}_{2} \cdot \mathbf{H}^{T}\right) \cdot\left(\boldsymbol{b} \cdot \mathbf{H}^{T}\right)\right] .
\end{aligned}
$$

As well the strong ellipticity condition these relations can be also written in a more compact form

$$
\mathbb{A}(\boldsymbol{\nu}) \cdot \boldsymbol{\xi}=\rho V^{2} \mathbb{B} \cdot \boldsymbol{\xi}
$$

where the matrix $\mathbb{B}$ is given by

$$
\mathbb{B}=\left[\begin{array}{cc}
\mathbf{I} & \mathbf{H} \cdot \Theta_{1}^{T} \cdot \mathbf{H}^{T} \\
\mathbf{H} \cdot \Theta_{1} \cdot \mathbf{H}^{T} & \mathbf{H} \cdot \boldsymbol{\Theta}_{2} \cdot \mathbf{H}^{T}
\end{array}\right] .
$$

Thus, the problem of acceleration wave propagation in the shell has been reduced to the spectral problem given by the algebraic equations (23). Owing to the existence of the potential-energy function $W, \mathbb{A}(\boldsymbol{\nu})$ is symmetric. Matrix $\mathbb{B}$ is also symmetric and positive definite. This property enables one to formulate an analogue of the Fresnel-Hadamard-Duhem theorem for the elastic shell: The squares of the velocities of second order singular curve (acceleration waves) in the elastic shells are real for arbitrary propagation directions specified by the vector $\nu$.

Note that the positive definiteness of $\mathbb{A}(\boldsymbol{\nu})$, which is necessary and sufficient for the wave velocity $V$ to be real, coincides with the strong ellipticity inequality (19).

\section{Conclusion}

For the nonlinear six-parametric theory of elastic shells we formulate two constitutive inequalities. The first one is analogous to Coleman-Noll inequality (the GCN-condition) in 3D nonlinear elasticity while the second is the strong ellipticity condition. We show that Coleman-Noll inequality is more restrictive and implies the strong ellipticity of the shell equilibrium equations. We also investigate the conditions of existence of accelerations waves in shell and shown that this conditions is equivalent to the strong ellipticity inequality. 
Acknowledgements The first author was partially supported by the Russian Science Support Foundation.

\section{References}

[1] C. Truesdell and W. Noll, The Nonlinear Field Theories of Mechanics, 3rd ed. (Springer-Verlag, Berlin, 2004).

[2] C. Truesdell, A First Course in Rational Continuum Mechanics (Academic Press, New York, 1977).

[3] E. Cosserat and F. Cosserat, Théorie des corps deformables (Herman et Flis, Paris, 1909). English translation: NASA TT F-11, 561 (NASA, Washington D.C., 1968).

[4] H. Altenbach and P.A. Zhilin, Adv. in Mech. 11, 107-148 (1988).

[5] H. Altenbach and P.A. Zhilin, in: Theories of Plates and Shells: Critical Review and New Applications, edited by R. Kienzler, H. Altenbach, and I. Ott (Springer-Verlag, Berlin, 2004), pp. 1-12.

[6] S.S. Antman, Nonlinear Problems of Elasticity (Springer-Verlag, New York et al., 1995).

[7] V.A. Eremeyev, in: Shell Structures: Theory and Applications, edited by W. Pietraszkiewicz and C. Szymczak (Taylor \& Francis, London et al., 2005), pp. 11-18.

[8] V.A. Eremeyev and L.M. Zubov, Izvestiya VUZov. Sev.-Kakavk. Region. Estestv. nauki. Special issue. Nonlinear Problems of Continuum Mechanics, 124-169 (2003).

[9] L. I. Shkutin, Mechanics of Flexible Bodies (in Russian). (Nauka, Novosibirsk, 1988).

[10] P. A. Zhilin, Int. J. Solids Struct. 12, 635-648 (1976).

[11] P.A. Zhilin, Dinamika i Prochnost' Mashin, Trudy LPI 386, 29-46 (1982).

[12] L. M. Zubov, Nonlinear Theory of Dislocations and Disclinations in Elastic Bodies (Springer-Verlag, Berlin et al., 1997).

[13] J. Chróścielewski, J. Makowski, and W. Pietraszkiewicz, Statics and Dynamics of Multifold Shells: Nonlinear Theory and Finite Element Method (in Polish) (Wydawnictwo IPPT PAN, Warszawa, 2004).

[14] A. Libai and J. G. Simmonds, Adv. Appl. Mech. 23, 271-371 (1998).

[15] A. Libai and J. G. Simmonds, The Nonlinear Theory of Elastic Shells, 2nd ed. (University Press, Cambridge, 1998).

[16] W. Pietraszkiewicz, Adv. Mech. 12(1), 51-130 (1989).

[17] V.A. Eremeyev and W. Pietraszkiewicz, J. Elast. 85, 125-152 (2006).

[18] C. Sansour and H. Bednarczyk, Computer Methods Appl. Mech. Eng. 120, 1-32 (1995).

[19] H. Altenbach, K. Naumenko, and P.A. Zhilin, in: Shell Structures: Theory and Applications, edited by W. Pietraszkiewicz and C. Szymczak (Taylor \& Francis, London et al. 2005), pp. 87-90.

[20] A. I. Lurie, Nonlinear Theory of Elasticity (in Russian) (Nauka, Moscow, 1980).

[21] V.A. Eremeyev and L. M. Zubov, Izv. AN SSSR. Mekhanika tverd. tela (Prxoc. USSR Academy of Science. Mech. solids) 3, 181-190 (1994).

[22] J.-L. Lions and E. Magenes, Problèmes aux limites non homogènes et applications (Dunod, Paris, 1968).

[23] G. Fichera, Existence Theorems in Elasticity (Springer-Verlag, Berlin et al., 1972.)

[24] V.A. Eremeyev, Doklady Physics 50(4), 204-206 (2005). 